\title{
Effect of Adrenocorticotropic Hormone and Corticosteroid on Release of Catecholamine from Adrenal Medulla
}

\author{
Minoru INABA, ${ }^{*}$ Kunie KAMATA,* \\ HIROAKI OKAWA, AND TAKESHI NAKAO \\ Division of Biological Function Research, Laboratories for \\ Bio-Medical Experimentation* and Department of Pharmacology, \\ Jikei University School of Medicine, Minato-ku, Tokyo
}

\begin{abstract}
Synopsis
A chemical method for the determination of catecholamine (CA) by pipsylation, reaction with p-iodobenzenesulfonyl chloride, was used to study CA release from the rat adrenal medulla or adrenal in vitro, and to estimate CA concentration in the rat adrenal medulla.

Removal of the rat pituitary gland caused a decrease of adrenal medulla weight within 7 days after hypophysectomy, and the decrease of medulla weight was accompanied with an elevation of CA concentration in medulla.

Corticosterone stimulated to increase CA release from adrenal medulla in vitro, but adrenocorticotropic hormone did not have a significant effect on CA release from adrenal medulla in vitro.
\end{abstract}

Almost all mammalian adrenals consist of two different tissues: a medulla which is a mass of chromaffin cells and a cortex which covers the former. It is well known that both the cortex and medulla respond quickly and simultaneously to the stress to secrete corticosteroid and catecholamine (CA) respectively. Although a functional relationship between these two neighboring tissues was suggested (West, 1954), details of the correlation are still obscure. Activity of phenylethanolamine-Nmethyltransferase, which catalyzes formation of adrenaline from noradrenaline in medulla, was proposed to be under a direct control of glucocorticoid produced by cortex (Wurtman and Axelrod, 1966), and this was confirmed recently (Kimura, 1968).

Catecholamine reacts with p-iodobenzenesulfonyl chloride (pipsyl chloride) under an appropriate condition to form the pipsyl ester which has an ultraviolet light absorption $(\lambda \mathrm{MeOH} 251 \mathrm{~m} \mu)$ (Inaba and Nakao, 1968).

Received for publication March 2, 1970.
The reaction is applicable to the determination of CA concentration in the rat adrenal or adrenal medulla as well as in the medium in which these tissues have been incubated. In the present paper the method for CA determination in biological materials such as the rat adrenal medulla or adrenal and the incubation medium of these tissues will be described. Using the method, effects of adrenocorticotropic hormone (ACTH) and corticosteroid on release of adrenal $\mathrm{CA}$ into the incubation media as well as a change of CA concentration in adrenal medulla after the removal of pituitary gland are studied.

\section{Materials and Methods}

Male albino rats (Donryu) weighing 200-380 g were used throughout the investigations. They were housed in groups of 4 or 5 in an air-conditioned room $\left(23^{\circ}-24^{\circ} \mathrm{C}\right)$ illuminated from 9 a.m. to 7 p.m., and were given food and water ad libitum. All animals were held in the conditioned room for about a month prior to being used. To minimize variation due to a circadian 
rhythmicity of adrenal CA content (Nakao and Hiraga, 1968), all animals were decapitated for experimental use at the definite time between $10 \mathrm{a}$. m. and 11 a.m. Hypophysectomy was performed by a parapharyngeal approach method under pentobarbital anesthesia (Nakao, 1955), and the completeness for removal of pituitary gland was checked by autopsy.

Adrenals were removed, placed on filter paper moistened with physioligical saline as soon as possible after decapitation of the animals, carefully freed of adhering fat with a pair of fine scissors, and weighed on a torsion balance. Each adrenal was divided into halves. Cutting adrenal in half should be as even as possible; 4 adrenal-halves of almost equal size can be obtained from a pair of adrenals after a little practice. Cortical tissue was then carefully dissected away with a pair of fine scissors to obtain adrenal hemi-medulla.

The 16 or 20 medulla-halves from 4 or 5 rats were distributed into the 2 lots in a humidor, each lot containing 8 or 10 halves. Each group of halves was weighed on a torsion balance and placed in a small flask containing 1.0 or $2.0 \mathrm{~m} l$ incubation medium. In this manner 2 flasks containing the equal numbers of medulla-halves of almost equal weight could be prepared. At the same time a blank flask containing 1.0 or $2.0 \mathrm{~m} l$ incubation medium alone was prepared. In some experiments the whole adrenal-halves were used instead of medulla-halves for incubation.

Flasks were placed in an incubator and shaken with constant speed at $36^{\circ}-37^{\circ} \mathrm{C}$ in air for $30 \mathrm{~min}$ or $1 \mathrm{hr}$. Medium used for incubation was Tris buffer solution, pH 6.0-6.8, containing $\mathrm{NaCl}(0.9 \%), \mathrm{KCl}(0.04 \%)$ and $\mathrm{CaCl}_{2}(0.02 \%)$. Krebs-Ringer bicarbonate buffer solution, $\mathrm{pH} 7.4$, was used in some experiments as incubation medium in the gas mixture of $95 \% \mathrm{O}_{2}$ and $5 \%$ $\mathrm{CO}_{2}$.

At the end of the incubation period, 2 aliquots of $0.4 \mathrm{~m} l$ incubation medium were transferred into $10 \mathrm{~m} l$ conical tubes. In order to exclude the trace amounts of corticosteroid which might be released from residual cortical tissue left on the medulla-halves, the medium was washed with $2 \mathrm{~m} l$ dichloromethane twice. Following centrifugation $0.2 \mathrm{~m} l$ aliquot of the extracted medium was transferred into a fresh conical tube and $0.05 \mathrm{~m} l$ glacial acetic acid was added. Pipsyl chloride synthetized in the laboratory, $100 \mu \mathrm{g}$ in $0.1 \mathrm{~m} l$ acetone, and $0.1 \mathrm{ml}$ of $10 \% \mathrm{Na}_{2} \mathrm{CO}_{3}$ were then pipetted into the tube, and the pipsylation reaction was carried out according to the method described previously for the pipsylation of estrogens (Inaba, Nakao and Kamata, 1967), except that dichloromethane was used for extraction of pipsylated product instead of chloroform. Dichloromethane extract was dried and dissolved in $1.5 \mathrm{ml}$ methanol for spectrometric analysis.

Medulla-halves in each flask were picked up on filter paper as soon as possible after the end of incuba- tion, washed with saline, blotted, weighed, and homogenized in $2.0 \mathrm{~m} l$ of cold $0.4 \mathrm{~N}$ perchloric acid followed by centrifugation to obtain the clear extract of medullary CA. 2 aliquots of $0.2 \mathrm{~m} l$ perchloric acid extract were taken into conical tubes and pipsylation reaction was carried out as described above.

The 2 known amounts of the standard adrenaline (1-Adrenaline, E. Merck), 2.5 and $5.0 \mu \mathrm{g}$, in $0.2 \mathrm{~m} l$ of $0.4 \mathrm{~N}$ perchloric acid were pipsylated in parallel with the incubation media and perchloric acid extracts of adrenal medulla.

Methanol solutions of the compound obtained by pipsylation of biological materials and the standard adrenaline were read their optical densities in a spectrophotometer (Hitachi) at $251 \mathrm{~m} \mu$. The amount of pipsylated compound was expressed as the amount of the standard adrenaline.

\section{Results}

Product of adrenaline or noradrenaline obtained by reaction with pipsyl chloride showed an ultraviolet light (UV) absorption curve with a single peak at $251 \mathrm{~m} \mu$ in methanol. UV absorption curves obtained by pipsylation of incubation media of medulla-halves coincided with that of adrenaline pipsylated (Fig. 1). Reaction product of the perchloric acid extract of medulla as well as the incubation medium of adrenal-halves also showed the same shape of UV absorption curve as that of adrenaline. A quantitative relationship was established between the amounts and the optical densities at $251 \mathrm{~m} \mu$ of adrenaline pipsylated (Fig. 2). In order to examine whether adrenaline in the incubation medium of medullahalves reacts quantitatively with pipsyl chloride, the 3 different samples for pipsylation were prepared in 3 conical tubes, each of which contained $0.2 \mathrm{ml}$ aliquot of $2 \mathrm{ml}$ incubation medium of 8 medulla-halves. Adrenaline${ }^{3} \mathrm{H}, 1,080 \mathrm{cpm}, 2,150 \mathrm{cpm}$ and $4,300 \mathrm{cpm}$ was added into the respective conical tube, then pipsylation reaction was carried out, followed by the counting of ${ }^{3} \mathrm{H}$ in the reaction products. The same experiment was repeated in triplicate. Adrenaline- ${ }^{3} \mathrm{H}$ (dl-adrenaline- $7-{ }^{3} \mathrm{H}, 3$ $\mathrm{Ci} / \mathrm{m} M$ ), purchased from C. E. N., Dept. des Radioisotopes, Mol., Belgium, was purified by 


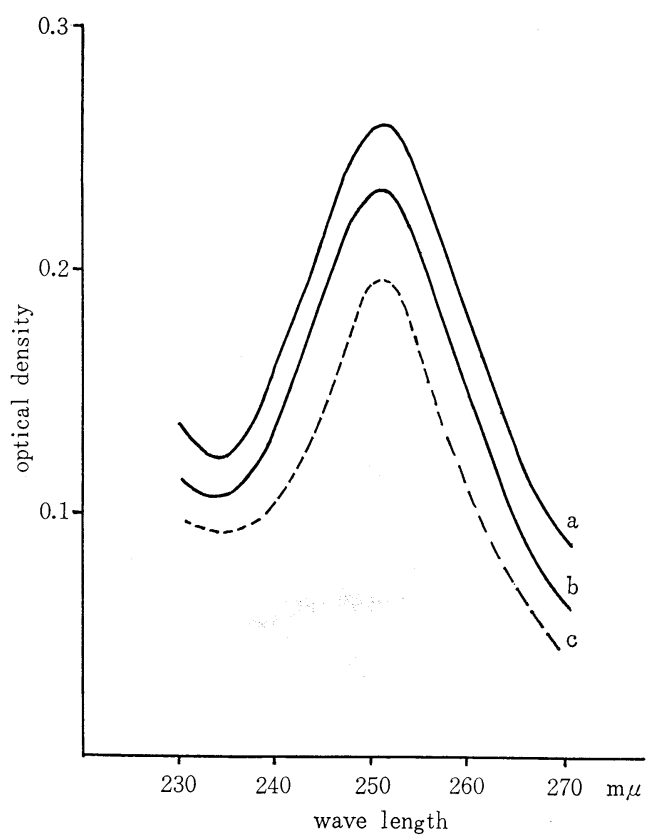

Fig. 1. Ultraviolet light absorption curves of pipsylated product of incubation media of rat adrenal medullae and of the standard adrenaline.

a. Absorption curve of the pipsylated product of medium in which adrenal medulla-halves were incubated for $30 \mathrm{~min}$.

b. The same as a, obtained by the successive 30 min-incubation.

c. Absorption curve of pipsylated product of the standard adrenaline.

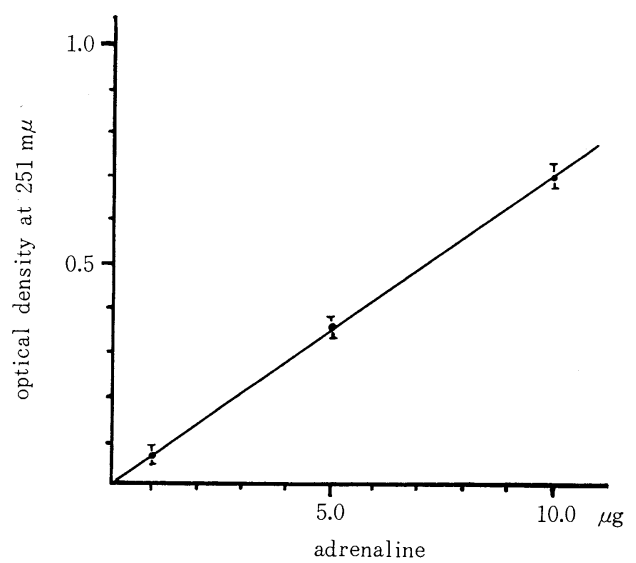

Fig. 2. Dose-absorption relationship of the standard adrenaline pipsylated.
Table 1. Quantitative pipsylation of adrenaline in the incubation medium of rat adrenal medullahalves

\begin{tabular}{|c|c|c|}
\hline $\begin{array}{l}\text { Sample for } \\
\text { pipsylation }\end{array}$ & $\begin{array}{c}{ }^{3} \mathrm{H}^{*}(\mathrm{cpm}) \text { added } \\
\text { to sample }\end{array}$ & $\begin{array}{c}{ }^{3} \mathrm{H}^{*}(\mathrm{cpm}) \text { found } \\
\text { in pipsylated } \\
\text { product of sample }\end{array}$ \\
\hline \multirow{3}{*}{$\begin{array}{l}\text { I. } 0.2 \mathrm{~m} l \text { alquot of } \\
2 \mathrm{~m} l \text { incubation } \\
\text { medium }\end{array}$} & 1,080 & 320 \\
\hline & " & 345 \\
\hline & 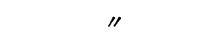 & 390 \\
\hline \multirow[t]{3}{*}{ II. Ibid. } & 2,150 & 640 \\
\hline & "I & 695 \\
\hline & " & 720 \\
\hline \multirow[t]{3}{*}{ III. Ibid. } & 4,300 & 1,250 \\
\hline & " & 1,220 \\
\hline & $" \prime$ & 1,310 \\
\hline
\end{tabular}

* Adrenaline $-7-{ }^{3} \mathrm{H}$

Sample for pipsylation I, II and III consisted of 3 conical tubes respectively, each of which contained $0.2 \mathrm{~m} l$ aliquot of $2 \mathrm{~m} l$ incubation medium of 8 adrenal medulla-halves and the known amount of adrenaline $-{ }^{3} \mathrm{H}$.

absorbing to alumina, followed by elution with $0.4 \mathrm{~N}$ perchloric acid before use. Reaction product containing adrenaline- ${ }^{3} \mathrm{H}$ pipsylated was dissolved in $10 \mathrm{~m} l$ of PPO-POPOPtoluene scintillator fluid, and the radioactivity was counted in a liquid scintillation spectrometer (Packard Tri-Carb Model 3002). The counting efficiency for ${ }^{3} \mathrm{H}$ was $30 \%$. As in Table 1, a quantitative relation was observed between the radioactivity in the reaction product and the amount of adrenaline $-{ }^{3} \mathrm{H}$ added.

Product obtained by pipsylation of media from several incubations of medulla-halves was developed by thin layer chromatography on silica gel (Kieselgel DF-5, Camag) in the solvent system of ligroine: ethyl acetate: chloroform (1: 4: 4 by vols.). UV absorbing compound which corresponded to the reference adrenaline pipsylated on chromatogram was extracted with the solvent mixture of methanol and dichloromethane (1: 1 by vol.). Dried extract was dissolved in methanol and the amount of pipsylated compound was quantified by UV absorption at $251 \mathrm{~m} \mu$, then added the known amount of adrenaline- ${ }^{3} \mathrm{H}$ pipsylated $(4,290 \mathrm{cpm})$. Methanol was evaporated and the dried sample was developed by thin 
Table 2. Specific radioactivity in pipsylated product of incubation medium of adrenal medulla-halves obtained during the purification steps by 3 different solvent systems of thin layer chromatography

\begin{tabular}{ccccc}
\hline \hline & $\begin{array}{c}\text { UV } \\
\text { Absorbing } \\
\text { compound } \\
(\mu \mathrm{g})\end{array}$ & $\begin{array}{c}\text { Radio- } \\
\text { activity } \\
(\mathrm{cpm})\end{array}$ & $\begin{array}{c}\text { Specific } \\
\text { radio- } \\
\text { activity } \\
(\mathrm{cpm} / \mu \mathrm{g})\end{array}$ \\
\hline $\begin{array}{c}\text { Before } \\
\text { After }\end{array}$ & Chromatography & 18.4 & 4,292 & 233 \\
1 st & Chromatography & 8.2 & 2,328 & 284 \\
2 nd & " & 6.9 & 1,944 & 282 \\
3 rd & " & 2.8 & 777 & 278 \\
\hline
\end{tabular}

Solvent systems used for thin layer chromatography and $\mathrm{R}_{f}$ values of $\mathrm{UV}$ absorbing compound on chromatogram were as follows: 1st chromatography-ligroine: ethyl acetate: chloroform (1: $4: 4$ by vols.), $\mathrm{R}_{f} 0.71$, 2nd chromatographybenzene: ethyl acetate: chloroform $(2: 2: 1$ by vols.), $\mathbf{R}_{f} 0.74$, 3rd chromatography-toluene: ethyl acetate: chloroform (4:1:2 by vols.), $\mathrm{R}_{f}$ 0.43 .

layer chromatography in succession in the 3 different solvent systems. The UV absorbing compound was eluted from each chromatogram, and the amount and radioactivity involved in the eluates were determined to obtain the specific radioactivity $(\mathrm{cpm} / \mu \mathrm{g})$. As in Table 2, the pipsylated compound of incubation media corresponding to adrenaline pipsylated on the chromatograms possessed the fairly constant specific radioactivities during the purifications with thin layer chromatography in the 3 different solvent systems, indicating that the pipsylated product of incubation media of rat adrenal medulla-halves contained mainly adrenaline pipsylated.

By using UV scanner (Mineralight, $2537 \AA$ ), the location of reaction product was clearly detected as UV absorbing spot on silica gel in thin layer chromatography as well as on paper (Whatman No. 1) in paper chromatography. The minimal amount of adrenaline pipsylated for the detection of its location on thin layer chromatogram was about $2.5 \mu \mathrm{g}$. Table 3 summarized the migration rates $\left(\mathrm{R}_{f}\right)$ of adrenaline pipsylated and noradrenaline pipsylated in the
Table 3. Migration rates ( $\mathbf{R}_{f}$ values) of adrenaline pipsylated and noradrenaline pipsylated in the various solvent systems of thin layer and paper chromatography

\begin{tabular}{|c|c|c|}
\hline $\begin{array}{l}\text { Solvent system of thin } \\
\text { layer chromatography }\end{array}$ & $\begin{array}{l}\text { Adrenaline } \\
\text { pipsylated }\end{array}$ & $\begin{array}{l}\text { Noradrenaline } \\
\text { pipsylated }\end{array}$ \\
\hline $\begin{array}{l}\text { Ligroine: ethyl acetate: } \\
\text { chloroform }(4: 2: 4 \text { by ve }\end{array}$ & ols.) & on origin \\
\hline Ibid. (2: $4: 3$ by vols.) & 0.54 & 0.42 \\
\hline Ibid. (1: $4: 4$ by vols.) & 0.65 & 0.52 \\
\hline $\begin{array}{l}\text { Chloroform: methanol } \\
\text { (9:1 by vol.) }\end{array}$ & 0.71 & 0.60 \\
\hline $\begin{array}{l}\text { Benzene: acetone } \\
\text { (1:1 by vol.) }\end{array}$ & 0.66 & 0.63 \\
\hline $\begin{array}{c}\text { Solvent system of } \\
\text { paper chromatography }\end{array}$ & $\begin{array}{l}\text { Adrenaline } \\
\text { pipsylated }\end{array}$ & $\begin{array}{c}\text { Noradrenaline } \\
\text { pipsylated }\end{array}$ \\
\hline $\begin{array}{l}\text { Ligroine: methanol: wate } \\
\text { (10: } 8: 2 \text { by vols.) }\end{array}$ & 0.07 & on origin \\
\hline $\begin{array}{l}\text { Toluene: ligroine: } \\
\text { methanol: water ( } 67: 33 \\
60: 40 \text { by vols.) }\end{array}$ & 0.42 & 0.11 \\
\hline $\begin{array}{l}\text { Benzene: ligroine: } \\
\text { methanol: water ( } 33: 67 \\
80: 20 \text { by vols.) }\end{array}$ & 0.64 & 0.38 \\
\hline
\end{tabular}

several solvent systems of thin layer and paper chromatography. Migration rates of the pipsylated products of incubation media and perchloric acid extracts of adrenal medulla-halves coincided to the reference adrenaline pipsylated in all solvent systems studied. Even when the reaction product was exposed to air at room temperature for a long period, such as 1 week, its migration rate and amount were not changed, indicating the stability of the reaction product. When the reaction product was partitioned between water and chloroform, it stayed mostly in the chloroform phase. In regard to the solubility of the pipsylated compound, it was dissolved in ethanol, methanol, acetone, toluene, chloroform, and dichloromethane.

A half section of the right adrenal of a normal rat was placed together with a half of the left adrenal in a flask containing the medium. In this manner 2 flasks containing 10 adrenalhalves from 5 normal rats and $2.0 \mathrm{ml}$ incubation medium (Tris buffer solution containing $\mathrm{NaCl}, \mathrm{KCl}$ and $\mathrm{CaCl}_{2}$ described in Materials and Methods) respectively, and 2 flasks con- 
taining medium alone were prepared, and the incubation was carried out for $30 \mathrm{~min}$. The amount of CA released into medium was compared between 2 flasks containing almost equal weights of adrenal-halves (Pool $\mathrm{A}$ and B). As illustrated in Figure 3, a quite good agreement of the amounts of CA released into medium was observed between Pool A and B in the separate 4 experiments.

Changes in weight and CA concentration of adrenal medulla due to hypophysectomy were investigated. In Table 4, changes in weight of

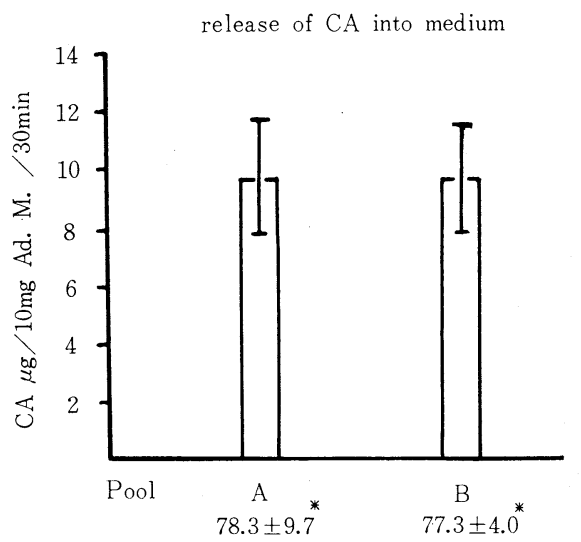

Number of rats used: 20

* Weight of adrenal-halves incubated, mean \pm S.D. (mg/flask)

See the text about Pool A and B,

Ad. M.: Adrenal medulla, CA: Catecholamine

Fig. 3. Comparison of the amounts of $\mathrm{CA}$ released from adrenal into incubation medium between 2 pools of almost equal weight of adrenal-halves. the whole body, adrenal and adrenal medulla as well as change of medullary CA concentration due to hypophysectomy were summarized. On the 5th day after hypophysectomy a significant reduction in weight of adrenals as well as of adrenal medullae was observed, accompanied with a slight increase of medullary CA concentration. On the 7 th day the reduction in weight became much more distinct and the medullary CA concentration was found to be around twice as much as that of the control.

Direct influence of ACTH on CA release from adrenal medulla was examined. The 8 adrenal medulla-halves, $9.3 \mathrm{mg}$, from 4 normal rats were incubated for $1 \mathrm{hr}$ in a flask containing $1 \mathrm{U}$. of ACTH (Schering) dissolved in 1.0 $\mathrm{m} l$ of Krebs-Ringer bicarbonate buffer solution, and the other 8 medulla-halves, $9.1 \mathrm{mg}$, were simultaneously incubated for $1 \mathrm{hr}$ without addition of ACTH. At the end of incubation, $0.4 \mathrm{~m} l$ aliquots of incubation medium were transferred in conical tubes from each flask for pipsylation. Tissues were then picked out, rinsed and incubated in the fresh medium for another $1 \mathrm{hr}$ in the same way as in the 1st $1 \mathrm{hr}$ incubation. The 4 experiments were repeated with 16 normal rats in total. As shown in Figure 4, no effect of ACTH on CA release from adrenal medulla was observed within $1 \mathrm{hr}$ of incubation. Although a tendency of suppression of CA release from adrenal medulla by ACTH addition was observed in the suc-

Table 4. Changes in weight of the whole body, adrenal and adrenal medulla, and in concentration of catecholamine after hypophysectomy

\begin{tabular}{ccccc}
\hline \hline $\begin{array}{c}\text { Hr after } \\
\text { hypophysectomy }\end{array}$ & $\begin{array}{c}\text { Body weight } \\
(\mathrm{g})\end{array}$ & $\begin{array}{c}\text { Weight of } \\
\text { an adrenal } \\
(\mathrm{mg})\end{array}$ & $\begin{array}{c}\text { Weight of a pair } \\
\text { of adrenal medulla } \\
(\mathrm{mg})\end{array}$ & $\begin{array}{c}\text { CA concentration } \\
\text { in adrenal medulla } \\
(\mu \mathrm{g} / 10 \mathrm{mg} \text { Ad. M.) }\end{array}$ \\
\hline -(Sham op.) & $378 \pm 10.6^{* *}$ & $20.2 \pm 2.6^{* *}$ & $4.60 \pm 0.53^{*}$ & 46.16 \\
$120 \mathrm{hr}$ (5 days) & $282 \pm 13.1$ & $12.9 \pm 1.1$ & $3.73 \pm 0.21$ & 53.75 \\
- (Sham op.) & $364 \pm 28.0^{* *}$ & $24.3 \pm 8.0^{* *}$ & $5.00 \pm 1.00^{*}$ & 42.33 \\
168 hr (7 days) & $292 \pm 19.4$ & $9.0 \pm 3.3$ & $3.07 \pm 0.12$ & 79.24 \\
\hline
\end{tabular}

In one experiment, 6 rats with the same birth date were divided in 2 groups. The 3 rats of one group were hypophysectomized and the 3 other rats were sham-operated as a control. Data were expressed as mean \pm standard deviation.

* $\mathrm{p}<0.05$ differs from hypophysectomy.

** $\mathrm{p}<0.01$ differs from hypophysectomy. 
cessive $1 \mathrm{hr}$ incubation, the difference in the amount of CA released into medium between ACTH addition and control was not statistically significant.

Influence of corticosteroid on CA release from adrenal medulla was examined in vitro.

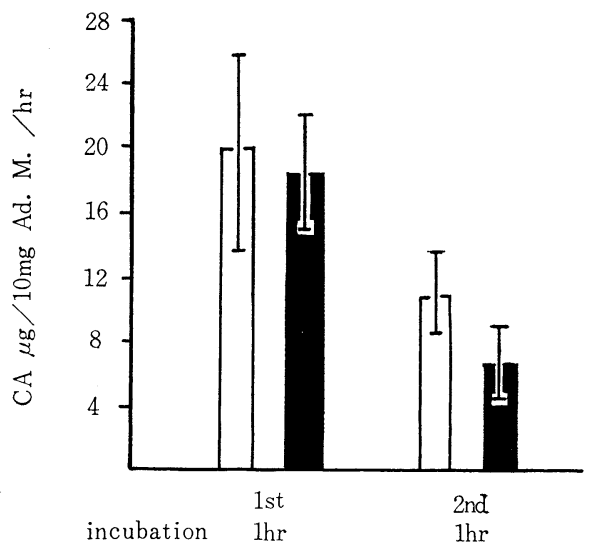

Fig. 4. Direct influence of adrenocorticotropic hormone on release of catecholamine from rat adrenal medulla.

The 4 normal rats were used in one experiment, and 4 experiments were repeated with 16 rats in total. Weights of adrenal medulla-halves incubated in Krebs-Ringer bicarbonate buffer solution were $9.1 \pm 2.0 \mathrm{mg}$ per flask for the control incubation $\square$, and $9.3 \pm 3.5 \mathrm{mg}$ per flask for the incubation with ACTH $\square$.

Table 5. Direct influence of corticosteroids on release of catecholamine from adrenal medulla

\begin{tabular}{clcc}
\hline $\begin{array}{c}\text { Number } \\
\text { of } \\
\text { rats used }\end{array}$ & $\begin{array}{c}\text { Steroid added } \\
\text { into medium }\end{array}$ & \multicolumn{2}{c}{$\begin{array}{c}\text { CA released into } \\
\text { medium } \\
(\mu \mathrm{g} / 10 \mathrm{mg} \mathrm{Ad} . M . / \mathrm{Hr})\end{array}$} \\
\hline 24 No addition (control) & $18.2 \pm 3.8^{*}$ \\
16 Desoxycorticosterone, & $0.29 \mathrm{mM}$ & $18.0 \pm 4.8$ \\
12 & Ibid. & $0.58 \mathrm{mM}$ & $26.0 \pm 8.4$ \\
12 Corticosterone, & $0.58 \mathrm{mM}$ & $38.7 \pm 1.5$ \\
\hline
\end{tabular}

Weight of medulla-halves incubated was $9.0 \pm$ $2.6 \mathrm{mg}$ per flask. Steroid used was dissolved in 0.1 $\mathrm{m} l$ of propylene glycol, then added into $0.9 \mathrm{~m} l$ of incubation medium. Control flask contained 0.1 $\mathrm{m} l$ of propylene glycol and $0.9 \mathrm{~m} l$ of incubation medium. Incubation medium was Tris buffer solution, $\mathrm{pH} 6.8$, containing $\mathrm{NaCl}, \mathrm{KCl}$ and $\mathrm{CaCl}_{2}$ as described in the Text. Incubation period of time was $1 \mathrm{hr}$. Data were expressed as mean \pm S.D.

* $\mathrm{P}<0.05$ differs from the corticosterone addition.
Adrenal medulla-halves, $9.0 \pm 2.6 \mathrm{mg}$, were incubated for $1 \mathrm{hr}$ in a flask containing $1.0 \mathrm{ml}$ incubation medium in which corticosteroid was dissolved. Corticosteroid was previously dissolved in $0.1 \mathrm{~m} l$ propylene glycol, then 0.9 $\mathrm{m} l$ incubation medium was added. Control flask contained $0.1 \mathrm{~m} l$ propylene glycol and $0.9 \mathrm{ml}$ incubation medium. Corticosteroids examined were corticosterone, $0.58 \mathrm{~m} M$, and desoxycorticosterone, 0.29 and $0.58 \mathrm{mM}$. As summarized in Table 5, corticosterone caused a striking increase of $\mathrm{CA}$ release from adrenal medulla. Although it was not statistically significant, desoxycorticosterone $(0.58 \mathrm{~m} M)$ also stimulated to increase CA release from adrenal medulla.

\section{Discussion}

In the present paper we described a chemical method for the determination of rat adrenal catecholamine (CA) by pipsylation. It seems that the method will be useful in view of the facts that $\mathrm{CA}$ in the aqueous media such as the incubation medium and the perchloric acid extract of rat adrenal medulla or the whole adrenal was able to react quantitatively with p-iodobenzenesulfonyl chloride (pipsyl chloride), and that the reaction product of $\mathrm{CA}$ with pipsyl chloride was stable and could be quantified by its ultraviolet light (UV) absorption at $251 \mathrm{~m} \mu$ with relative ease. Moreover, the method will be more practical for the determination of rat adrenal CA than the fluorometric methods which are very popular at present. Although the method reported here is very precise, it is not so highly sensitive as to be able to be applied to the determination of CA in the body fluid or the tissues other than adrenal. It can possibly, however, be much improved by the use of radioactive pipsyl chloride as a reagent for pipsylation. While the pipsylation was applied to the determination of total CA in the adrenal or adrenal medulla, adrenaline pipsylated can be separated from noradrenaline pipsylated by thin layer or pa- 
per chromatography, suggesting that the determination of an individual CA can possibly be achieved. As mentioned by some investigators (Whitehead and Dean, 1968), the specificity of pipsylation reaction is not high. Compounds in the biological materials such as some amino acids and phenolic steroids react with pipsyl chloride. This point must be taken into consideration when the pipsylation reaction is applied to the biological materials and a careful examination for the specificity of the method should be carried out before the practical use of the method. In our experiments it is obvious that the reaction product obtained by pisylation of incubation media of rat adrenal medulla-halves contained adrenaline pipsylated mainly. This was proved by the same mobility of the reaction product as the standard adrenaline pipsylated in thin layer and paper chromatography, and by the constant specific radioactivities obtained during the purification of the reaction product mixed with adrenaline- ${ }^{3} \mathrm{H}$ pipsylated by thin layer chromatography. Molecular extinction coefficient of the adrenaline pipsylated in UV absorption could not be calculated because the chemical structure of pipsylated product is not identified at present, but it is supposedly near to those of the steroids having $\Delta^{4}$-3ketone group in their molecules. Sensitivity for the determination of CA pipsylated by UV absorption is around $0.5 \mu \mathrm{g}$.

Interestingly, glucocorticoid produced by rat adrenal cortex has been proposed to control directly the activity of phenylethanolamine-N-methyltransferase (PNMT) catalyzing the formation of adrenaline from noradrenaline in adrenal medulla on the basis of the fact that the decreased PNMT activity due to hypophysectomy recovered by the administration of glucocorticoid (Wurtman and Axelrod, 1966). They reported that the PNMT activity decreased $72.6 \%$ within $17-21$ days after hypophysectomy, but we learned in their paper that the adrenaline content in adrenal decreased only $23.5 \%$ within $17-21$ days after hypophysectomy. A change in weight of ad- renal medulla by hypophysectomy should be taken into consideration. The medulla weight, in fact, was reported to decrease significantly within 17-21 days after hypophysectomy (Mikulaskova-Rochova and Linet, 1967). We found that the weight of rat adrenal medulla decreased about $40 \%$ within less than 17-21 days, such as 7 days after hypophysectomy. From these points of view, it will be important to study a change of medullary CA concentration, not CA content, after hypophysectomy. The data presented here indicated that the medullary CA concentration was remarkably elevated within 7 days after hypophysectomy, and that corticosterone stimulated to increase CA release from adrenal medulla in vitro. From these findings, we concluded that the corticosteroid produced by adrenal cortex would act on adrenal medulla directly and stimulate not only PNMT activity but the release of CA. These observations would also suggest a possible influence of the pituitary-adrenocortical axis on CA release from adrenal medulla. Our findings differ from those of Schneider (1969), who concluded that there would be no differences in the mechanisms of CA secretion from the cortex-free perfused medulla and the perfused whole adrenal gland of bovine. The difference between those conclusions might be ascribed to the difference of procedures employed to the studies of CA release as well as to the different animal species used for experiments.

\section{Acknowledgements}

This study was supported in part by a grant from the Ministry of Education in Japan. The authors wish to thank Drs. K. Hiraga and S. Imai for providing the enviromentally controlled and hypophysectomized rats.

\section{References}

Inaba, M, T. Nakao and K. Kamata (1967). Endocrinol. Japon. 14, 357. 
Inaba, M. and T. Nakao (1968). Folia Pharmacol. Japon. 64, 8 .

Kimura, T. Proceedings XXXXI Annual Meeting of Japan Endocrinological Society, p 8 (1968).

Mikulaskova-Rochova, J. and O. Linet (1967). J. Endocrinol. 39, 611.

Nakao, T. Method of hypophysectomy (Parapharyngeal method) for rabbit, rat, guinea pig and hamster, Ito, Y., ed. Ishiyaku Book Publishing Co., Tokyo, p 147 (1955).

Nakao, T. and K. Hiraga. Proceedings XXXXI
Annual Meeting of Japan Endocrinolgical Society, p 11 (1968).

Schneider, F. H. (1969). Br. J. Pharmac. 37, 371.

West, G. B. (1954). Pharmacol. Rev. 6, 29.

Whitehead, J. K. and H. G. Dean. The isotope derivative method in biochemical analysis, in Method of biochemical analysis, Glick, D. ed., Interscience Publishers, New York, vol. 16, p 1 (1968).

Wurtman, R. J. and J. Axelrod (1966). J. Biol. Chem. 241, 2301. 